\title{
DNA Demethylation of the Foxp3 Enhancer Is Maintained through Modulation of Ten-Eleven- Translocation and DNA Methyltransferases
}

\author{
Varun Sasidharan Nair ${ }^{1}$, Mi Hye Song ${ }^{1}$, Myunggon $\mathrm{Ko}^{2}$, and Kwon $\mathrm{lk} \mathrm{Oh}^{1, *}$
}

\begin{abstract}
Stable expression of Foxp3 is ensured by demethylation of CpG motifs in the Foxp3 intronic element, the conserved non-coding sequence 2 (CNS2), which persists throughout the lifespan of regulatory $\mathrm{T}$ cells (Tregs). However, little is known about the mechanisms on how CNS2 demethylation is sustained. In this study, we found that Ten-ElevenTranslocation (Tet) DNA dioxygenase protects the CpG motifs of CNS2 from re-methylation by DNA methyltransferases (Dnmts) and prevents Tregs from losing Foxp3 expression under inflammatory conditions. Upon stimulation of Tregs by interleukin-6 (IL6), Dnmt1 was recruited to CNS2 and induced methylation, which was inhibited by Tet2 recruited by IL2. Tet2 prevented CNS2 re-methylation by not only the occupancy of the CNS2 locus but also by its enzymatic activity. These results show that the CNS2 methylation status is dynamically regulated by a balance between Tets and Dnmts which influences the expression of Foxp3 in Tregs.
\end{abstract}

\section{INTRODUCTION}

Forkhead box $\mathrm{P3}^{+}$(Foxp3 $^{+}$) $\mathrm{CD}^{+}$regulatory $\mathrm{T}$ cells (Tregs) control a variety of immune responses in a Foxp3 dependent manner (Fontenot et al., 2003; Hori et al., 2003). During these processes, Tregs differentiate functionally and adapt themselves to diverse environmental stimuli (Campbell and Koch, 2011). For example, some Tregs co-express Foxp3 and other lineage or tissue specific transcription factors like T-bet, ROR- $\gamma$ t, Bcl6 and PPAR- $\gamma$ to control the corresponding immune and non-immune responses (Burzyn et al., 2013; Chaudhry et al., 2009; Chung et al., 2011; Cipolletta et al., 2012; Koch et al., 2009; Linterman et al., 2011; Ohnmacht et al., 2015; Sefik et al., 2015; Wang et al., 2011). What is interesting is that some of the

${ }^{1}$ Department of Pathology, Hallym University College of Medicine, Chuncheon 24252, Korea, ${ }^{2}$ School of Life Sciences, Ulsan National Institute of Science and Technology, Ulsan 44949, Korea

${ }^{*}$ Correspondence: kwonik@ hallym.ac.kr

Received 15 November, 2016; accepted 24 November, 2016; published online 13 December, 2016

Keywords: cytokine, DNA demethylation, Foxp3, regulatory T cell, TenEleven-Translocation (Tet) lineage specifying transcription factors expressed together with Foxp3 like T-bet, GATA3, ROR- $\gamma \mathrm{t}$ and Bcl6 are induced by proinflammatory cytokines antagonizing Foxp3 expression. These reports suggest that unique mechanisms stabilizing the expression of Foxp3 should exist for Tregs to adapt to environmental changes and to preserve their identity at the same time (Sakaguchi et al., 2013).

Stable expression of Foxp3 is accompanied by epigenetic modulation of the CpG motifs within the evolutionarily conserved non-coding sequence 2 (CNS2) enhancer of the Foxp3 locus (Floess et al., 2007; Huehn and Beyer, 2015; Huehn et al., 2009; Kim and Leonard, 2007). Demethylation of the CpG motifs in the CNS2 enhancer enables critical transcription factors like Foxp3 and Runx1-Cbf- $\beta$ complex to access the CNS2 enhancer region and the CNS2 enhancer itself to interact with the promoter through a loop structure, which, as a result, has central roles in stabilizing Foxp3 expression especially under inflammatory conditions (Feng et al., 2014; Li et al., 2014; Zheng et al., 2010). Consistent with the above reports, CNS2 is demethylated in Tregs expressing Foxp3 stably but methylated in activated $\mathrm{CD}^{+} \mathrm{T}$ cells or TGF- $\beta$-induced Tregs expressing Foxp3 transiently (Miyao et al., 2012; Polansky et al., 2008).

CNS2 demethylation occurs in the thymus or periphery through iterative DNA oxidation reactions done by Ten-ElevenTranslocation (Tet) family members (Sasidharan Nair et al., 2016; Toker et al., 2013; Yang et al., 2015; Yue et al., 2016) and is known to be maintained consistently once established (Miyao et al., 2012). Indeed, CNS2 demethylation, instead of Foxp3 expression, was reported to be used as a reliable method for counting Tregs in peripheral blood and solid tissue (Wieczorek et al., 2009). However, how CNS2 demethylation is maintained in Tregs has not been clearly elucidated yet. In this study, we addressed the question whether Tet proteins have a role in maintaining as well as generating CNS2 demethylation and found that Tet proteins recruited to the CNS2 locus by IL2 protect the demethylated CpG motifs from being re-methylated by the occupancy of the CNS2 locus and its demethylase activity resulting in the stable expression of Foxp3 in Tregs.

\section{MATERIALS AND METHODS}

\section{Mice}

Wild-type (WT) C57BL/6 (B6) mice were purchased from Koatech (Pyeongtaek, Gyeongi-do, Korea). CD45.1 congenic (B6.SJL-Ptprc ${ }^{a}$ Pepc $^{b} /$ BoyJ), Foxp3-GFP transgenic (Foxp3 
bicistronic reporter mice expressing EGFP: B6.Cg-Foxp $\left.3^{t m 2 T c h} / J\right)$, floxed Tet2 transgenic $\left(\mathrm{B} 6 ; 129 \mathrm{~S}-\right.$ Tet2 $^{\text {tm1. }}{ }^{1 \text { laai }} / \mathrm{J}$, Tet2 ${ }^{\text {flit }}$ ) (MoranCrusio et al., 2011) mice were obtained from The Jackson Laboratory (Bar Harbor, ME). To generate Tet2 deficient mice (CD4-Cre $\times$ Tet2 $2^{\mathrm{fl} / \mathrm{fl}}$, referred to as Tet2 ${ }^{-/-}$mice), CD4-Cre transgenic mice were crossed to floxed Tet2 transgenic mice. The level of Tet2 expression was checked by RT-qPCR in CD4 ${ }^{+} \mathrm{T}$ cells and Tet2, but not Tet1 or Tet3, mRNA was greatly downregulated in $\mathrm{CD}^{+} \mathrm{T}$ cells in Tet2 ${ }^{-/-}$mice (data not shown). In most cases, Tet2 ${ }^{-/}$mice were further crossed with YFP reporter (floxed-STOP-YFP) mice (B6.129X1-Gt(ROSA)26Sor ${ }^{\text {m1(EYFP)Cos/ }}$ $\mathrm{J}$, Jackson laboratory) for checking the activity of the Cre enzyme. Tet $2^{\mathrm{fl} / \mathrm{fl}}$ or CD4-Cre $\times$ Tet ${ }^{+/+}$mice were used as WT control. OT-II transgenic mice expressing OVA-specific TCR were bred to Tet $^{-/-}$mice to generate OT-II/Tet2 ${ }^{-/-}$mice, which were used for the adoptive transfer followed by OVA/CFA immunization experiments. Foxp3-GFP-Cre transgenic mice (NOD/ShiLt$\mathrm{Tg}$ (Foxp3-EGFP/cre)1cJbs/J, Jackson laboratory, referred to as Foxp3-Cre mice) were also crossed with floxed Tet2 transgenic mice to delete Tet2 specifically in Tregs. Since we were not confident how many times Foxp3-Cre mice were backcrossed with B6 mice, Foxp3-Cre $\times$ Tet2 $2^{\text {flfll }}$ mice were considered in the mixed background $(B 6 \times N O D)$ and littermates were used as WT controls. Foxp3-Cre mice were used only in Figs. 2B, 2C and Supplementary Fig. S2. Only male mice were chosen in CNS2 demethylation analysis. All animal experimentations were conducted in accordance with guidelines and approval of the International Animal Care and Use Committees (IACUC) of Hallym University (Hallym 2014-74, 2015-63).

\section{Cell isolation and flow cytometry}

To sort naïve CD4 ${ }^{+}$cells and Treg cells, CD44, CD25 and YFP were used (naïve cells: $\mathrm{CD} 4^{+} \mathrm{CD} 8^{-} \mathrm{CD} 44^{\text {low }} \mathrm{CD} 25^{-} \mathrm{YFP}^{+}$; Treg cells: $\left.\mathrm{CD} 4^{+} \mathrm{CD} 8^{-} \mathrm{CD} 25^{+} \mathrm{YFP}^{+}\right)$. The post-sort purity for each cell type was usually $>95 \%$. We purchased the following monoclonal antibodies from BD Biosciences, eBioscience (USA) or BioLegend (USA) for flow cytometry: R-phycoerythrin (PE)-, PerCP-Cy5.5-, Allophycocyanin (APC)- or Brilliant Violet 421 (BV421)-labeled anti-CD25 (clones PC61 and 7D4), PE-Cy7-, PerCP-Cy5.5- or APC- or BV421-labeled anti-CD4 (clones RM4-5 and GK1.5), Fluorescein isothiocyanate (FITC)-, PE- or PerCP-Cy5.5-labeled anti-CD44 (clone IM7), PerCP-Cy5.5- or APC-labeled anti-CD45.1 (clone A20), PE- , PE-Cy7-, PerCPCy5.5- or Brilliant Violet 510 (BV510)-labeled-anti-CD45.2 (clone 104), PE-labeled-anti-CD62L (clone MEL-14), PElabeled-anti-CD69 (clone H1.2F3), PerCP-Cy5.5- or APC- or APC-Cy7-labeled-anti-CD8 (clone 53-6.7), APC-labeled-antiCD86 (clone GL1), PE-labeled-anti-CTLA4 (clone UC10-4F1011), Alexa Fluor 488- or PE-labeled-anti-Foxp3 (clone FJK-16s), APC-labeled-anti-IFN- $\gamma$ (clone XMG1.2), PE-labeled-IL17 (clone TC11-18H10.1) unconjugated anti-Myc (9E10, Santa Cruz Biotechnology, Dallas, Texas) and APC-labeled-donkey anti-mouse IgG (Jackson immunoresearch, West Grove, PA). Intracellular Foxp3, cytokines, myc-tagged proteins were stained using Foxp3 Staining Buffer set (eBioscience). For cytokine analysis, cells were cultured for 4 hours in the presence of PMA/ionomycin plus monensin (BD biosciences) before intracellular cytokine staining. Data were acquired through FACS Calibur or FACS Canto-II (BD Biosciences) and were analyzed with FlowJo software (Tree Star, USA) (Kim et al., 2015).

\section{Cell culture}

FACS-sorted cells were cultured in complete RPMI-1640 medium (WelGENE, Korea), supplemented with 10\% FBS (WelGENE), penicillin, streptomycin (Sigma-Aldrich, USA), L-glutamine (2 $\mathrm{mM}$; Life Technologies, USA), sodium pyruvate (2 mM; SigmaAldrich), non essential amino acid (0.1 mM; Sigma-Aldrich) and 2-ME (50 $\mu \mathrm{M}$; Sigma-Aldrich). For CD3/CD28 stimulation, FACS-sorted cells were stimulated by plate-bound anti-CD3 (2C11, $1 \mu \mathrm{g} / \mathrm{ml}$; eBioscience) plus CD28 $(37.51,1 \mu \mathrm{g} / \mathrm{ml})$ in the presence or absence of recombinant murine IL2 (rlL2; Peprotech, Rocky Hill, NJ, USA), recombinant IL6 (rlL6; Peprotech), recombinant IL4 (rIL4; Peprotech) or recombinant IL12 (rlL12; Peprotech) for indicated periods.

\section{Foxp3 demethylation analysis}

The genomic DNA was extracted from the FACS-sorted live cells by using the Blood \& Tissue Genomic DNA Extraction kit (Qiagen, USA). For isolation of DNA from cells fixed and stained with anti-Foxp3 mAb, we used the protocol described previously (Hansmann et al., 2010; Piper et al., 2014). Briefly, sorted cells $\left(\mathrm{CD} 4^{+} \mathrm{CD} 8^{-} \mathrm{Foxp} 3^{+} \mathrm{CD} 25^{+}\right)$were incubated with $300 \mu$ lysis buffer $(10 \mathrm{mM}$ Tris- $\mathrm{HCl}, 100 \mathrm{mM} \mathrm{NaCl}, 50 \mathrm{mM}$ EDTA, $0.5 \%$ SDS, $0.1 \mu \mathrm{g} / \mathrm{ml}$ proteinase $\mathrm{K}$, and $20 \mu \mathrm{g} / \mathrm{ml}$ RNase A) for 24 hours at $60^{\circ} \mathrm{C}$. Then DNA was extracted by phenol/chloroform/isoamyl alcohol solution (25:24:1) and precipitated overnight by ethanol. Extracted genomic DNAs were converted by the EZ DNA methylation gold kit (Zymo Research, USA). Anti-sense (Ohkura et al., 2012) strands of bisulfite-treated DNA were then subjected to PCR for amplification of CNS2 (12 CpG motifs were analyzed). The PCR products obtained were cloned into the pGemT-easy vector (Promega, USA) and 10-30 individual clones from each sample were sequenced with M13 reverse primer (GAAACAGCT ATGACCATG).

\section{Chromatin immunoprecipitation}

Nuclear lysate was sonicated to make small DNA fragments ranging from $100-500$ base pairs and then incubated with antiTet2 (ab94580, Abcam, UK) or anti-Dnmt1 Ab (H-300, Santa Cruz Biotechnology) overnight at $4^{\circ} \mathrm{C}$. Isotype-matched control $\mathrm{Ab}$ was used for the negative control. Immune complexes containing DNA fragments were precipitated using Dynabeads (Invitrogen, USA) or EZ-ChIP kit (Milipore, Germany). Relative enrichment of the target regions in the precipitated DNA fragments was analyzed by qPCR. The sequences of primers are as follows.

CNS2 forward, 5'-AAC CTT GGG CCC CTC TGG CA-3' CNS2 reverse, 5'-GGC CGG ATG CAT TGG GCT TCA-3'

Foxp3 promoter forward, 5'-CTT CTG GGA GCC AGC CAT-3'

Foxp3 promoter reverse, 5'-GCT GTA CTC CCC CCA CAA ATT-3'

\section{Quantitative RT-PCR (RT-qPCR)}

RNA was isolated from FACS-sorted cells using the RNeasy Mini kit (Qiagen) or Trizol (Life Technologies), and reversetranscribed into cDNA using QuantiTect Reverse Transcription kit (Qiagen). Quantitative PCR (qPCR) reactions were performed on RotorGene 6000 system (Qiagen) using AccuPower GreenStar qPCR kit (Bioneer, Korea). All data were normalized to actin. Non-specific amplification was checked by the use of melting curve and agarose gel electorphoresis. The sequences of primers are as follows.

Tet1 forward, 5'-GAG CCT GTT CCT CGA TGT GG-3' Tet1 reverse, 5'-CAA ACC CAC CTG AGG CTG TT-3' Tet2 forward, 5'-AGA GCC TCA AGC AAC CAA AA-3' Tet2 reverse, 5'-ACA TCC CTG AGA GCT CTT GC-3' Tet3 forward, 5'-TCC GGA TTG AGA AGG TCA TC-3' Tet3 reverse, 5'-CCA GGC CAG GAT CAA GAT AA-3' 


\section{Actin forward, 5'-CAT CCG TAA AGA CCT CTA TGC CAA C-3' Actin reverse, 5'-ATG GAG CCA CCG ATC CAC A-3'}

\section{Transfection}

Plasmids transfection experiments were performed using FACS-sorted Tet2 ${ }^{-1-}$ Tregs. Cells were transfected with plasmids encoding Myc-tagged mouse Tet2 (wt or mutant) or empty vector by using the electroporation kit (Amaxa Nucleofector Kit and Nucleofector device, Lonza, USA). Transfected Tregs were cultured for 3 days under the indicated condition for CNS2 demethylation study. Transfection efficiency and the expression levels were checked 4 hours after transfection by flow cytometry and RT-qPCR.

\section{Small interfering RNA transfection}

Transfection of Tregs was performed using the Mouse T Cell Nucleofector Kit and the Nucleofector device (X-001 program) according to the kit directions (Lonza). Cells were transfected with 300 pmol SMART pool siRNAs (Dharmacon, USA) designed against mouse Tet1 (M-062861-01-0005) and mouse Tet3 (L-054156-01-0005), or non-targeting control siRNAs (SN1001, Bioneer). The expression levels of each Tet were checked 4 hours after electroporation by RT-PCR. (Mantei et al., 2008).

\section{In vitro Suppression Assay}

FACS-sorted naïve $\mathrm{CD}^{+} \mathrm{T}$ cells labeled with cell trace violet (cell division dye, Life Technologies) were used as responders. Antigen presenting cells were prepared by depleting $\mathrm{CD} 4^{+}$and $\mathrm{CD}^{+}$cells from wild-type B6 splenocytes using flow cytometry. Responder cells $\left(5 \times 10^{3}\right)$ were cultured with antigen presenting cells $\left(2 \times 10^{4}\right)$ and soluble anti-CD3 $\mathrm{mAb}(0.3 \mu \mathrm{g} / \mathrm{ml})$ in the absence or presence of various numbers of Treg cells for 3 days. The division of responder cells was assessed by dilution of cell trace violet.

\section{Adoptive transfer and immunization experiments}

FACS-sorted OT-II Tregs were injected intravenously into congenic WT B6 mice. The next day, the recipient mice were subcutaneously immunized in the left flank with $100 \mu \mathrm{g}$ of OVA in CFA (Sigma-Aldrich) and injected intraperitoneally with sulfinpyrazone (10 $\mu \mathrm{g} / \mathrm{g}$ body weight) or vehicle (DMSO) every day. In 7 days OT-II CD4 ${ }^{+} \mathrm{Foxp}^{+}$Tregs of donor origin were FACSsorted from the draining left axillary LNs and subjected to CNS2 demethylation study. In some cases, the recipient mice were treated with congenically marked OT-II naïve and Treg cells together with anti-IL2 mAb.

\section{Statistical analyses}

A two-tailed, unpaired, student's $t$-test was used to calculate the statistical significance of differences between two groups unless specified. $\mathrm{P}$ values were represented as follows: ${ }^{\star \star \star}, P<$ $0.001 ;{ }^{*}, P<0.01$; ${ }^{*}, P<0.05$, whereas NS (not significant) was used to denote $P$ values $>0.05$. Error bars indicate s.e.m. ND means 'not detected'.

\section{RESULTS AND DISCUSSION}

Inflammatory cytokines cause CNS2 to be methylated in Tet2 $^{-/-}$Tregs

Previously, it was shown that Tet family members including Tet1, Tet2 and Tet3 have an essential role in demethylating the CpG motifs of the Foxp3 enhancer, CNS2, during the development of Tregs and contribute to the stable expression of Foxp3 (Yang et al., 2015; Yue et al., 2016). These reports led us to question how the established CNS2 demethylation is protected in mature Tregs where DNA methyltransferases (Dnmts) work actively (Wang et al., 2013) and whether Tet proteins might contribute by not only generating but also maintaining the CNS2 demethylation. To address these issues, the CNS2 methylation status of wild type (WT) and Tet2 deficient Tregs was investigated using the ovalbumin (OVA) immunization model. Tet2 deficient mice (referred to as Tet2 ${ }^{-/-}$mice) were generated by crossing floxed Tet2 (Tet2 $\left.{ }^{\mathrm{fl} / \mathrm{fl}}\right)$ with CD4-Cre transgenic mice. The amount of Tet2, but not Tet1 or Tet3, transcripts greatly decreased in the $\mathrm{CD} 4^{+} \mathrm{T}$ cells (data not shown). Tet $2^{-/-}$mice were further crossed with OT-II transgenic mice expressing OVA-specific TCR (OT-II/Tet2 ${ }^{-/-}$mice) for the OVA immunization experiments. Next, we isolated $\mathrm{CD}_{4}^{+} \mathrm{CD} 25^{+}$ Tregs from the LNs of the OT-IINT or OT-II/Tet2-/- mice (Supplementary Fig. S1) and injected them into congenically marked WT B6 mice. CNS2 among the three enhancers (CNS1, CNS2, and CNS3) of the Foxp3 gene contains 14 CpG motifs, and 12 $\mathrm{CpG}$ motifs in the antisense strands of the DNA were analyzed in this study (Fig. 1A). Freshly isolated OT-IINT and OTII/Tet2 $2^{-1-}$ Tregs, before being injected, had completely demethylated CpG motifs (Fig. 1B, left). The recipient mice were then immunized with OVA emulsified in complete Freund's adjuvant (CFA). CD4 ${ }^{+}$Foxp3 $^{+}$Tregs of donor origin in the draining LNs were re-sorted after 7 days and subjected to bisulfite sequencing to analyze the methylation status of the $\mathrm{CpG}$ motifs in the Foxp3 CNS2 region (referred to as CNS2 demethylation analysis hereafter). Almost all the CpG motifs in the CNS2 of the transferred WT Tregs maintained their demethylated status. In contrast, a significant number of $\mathrm{CpG}$ motifs became methylated in the CNS2 of the Tet2 ${ }^{-/-}$Tregs (Fig. 1B, right). Consistent with the results of the CNS2 demethylation analysis, the percentage of Foxp3-expressing cells of donor origin was reduced in the Tet2 ${ }^{-/-}$Tregs (Figs. 1B and 1D). Next, we additionally injected sulfinpyrazone or vehicle (DMSO) into the recipient mice treated as above (Fig. 1B). Because sulfinpyrazone is an inhibitor of sodium dependent vitamin $C$ transporters and can prevent vitamin $\mathrm{C}$ from entering cells and activating Tet proteins (Dickson et al., 2013), we expected that CNS demethylation might be reduced more in the Tet2 $2^{-/-}$Tregs treated with sulfinpyrazone simultaneously. The $\mathrm{CD}^{+} \mathrm{Foxp}^{+}$Tregs of donor origin were re-sorted and subjected to bisulfite sequencing after 7 days. As expected, a significant number of CpG motifs became methylated in the CNS2 of the Tet2 $2^{-/-}$Tregs treated with DMSO, and the extent of CNS2 methylation was larger in the Tet $2^{-/-}$Tregs treated with sulfinpyrazone (Fig. 1C). However, the expression of Foxp3 in the donor cells did not change significantly by the sulfinpyrazone treatment (Fig. 1D). We next performed the CNS2 demethylation analysis with Foxp3-Cre $\times$ Tet $2^{\mathrm{flft}}$ transgenic mice and found similar results (Supplementary Fig. S2). Taken together, these findings suggested that Foxp3 CNS2 demethylation and the stable expression of Foxp3 are actively maintained by Tet2 and its activator vitamin $C$ in Tregs.

DNA methylation is known to be caused by Dnmts, which led us to check whether Dnmts are involved in the CNS2 remethylation of Tet2 ${ }^{-/}$Tregs. OT-II/Tet2 ${ }^{-1-}$ Tregs were adoptively transferred into WT congenic mice, which were immunized with OVA/CFA and simultaneously treated with sulfinpyrazone, and a cohort of recipient mice were additionally injected with 5azacytidine, which is a cytosine nucleoside analogue that inhibits the function of Dnmts. Interestingly, CNS2 re-methylation was inhibited well by the 5-azacytidine treatment (Fig. 1E) supporting the essential role of Dnmts in CNS2 re-methylation induced by cognate antigens and inflammation. 
A

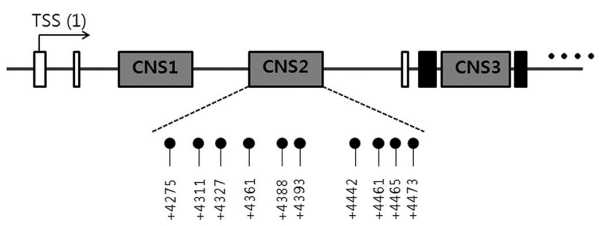

B

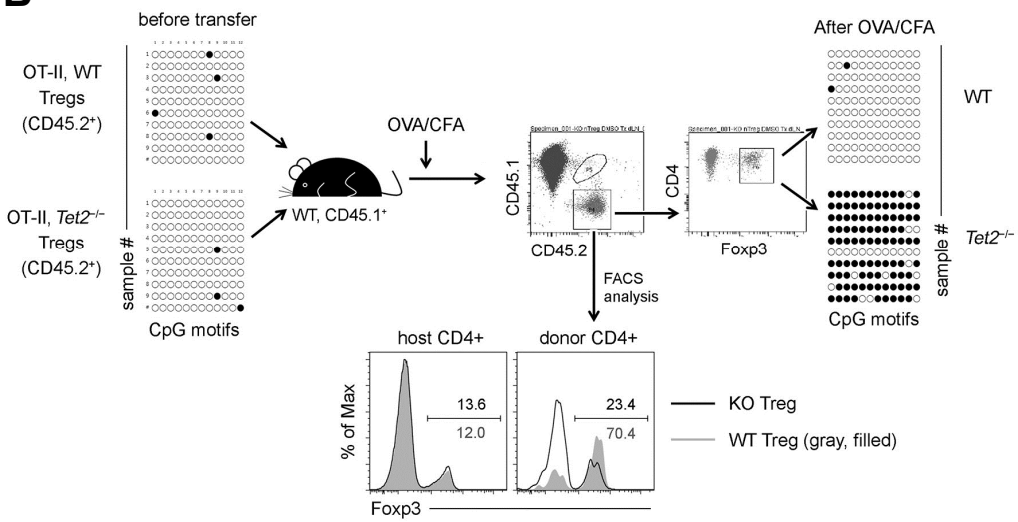

C

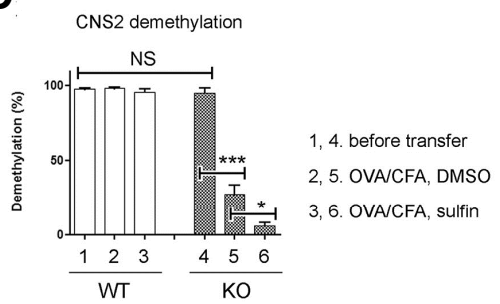

D

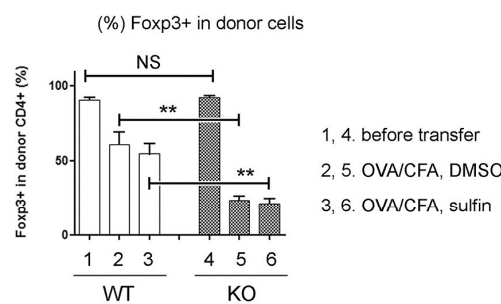

E
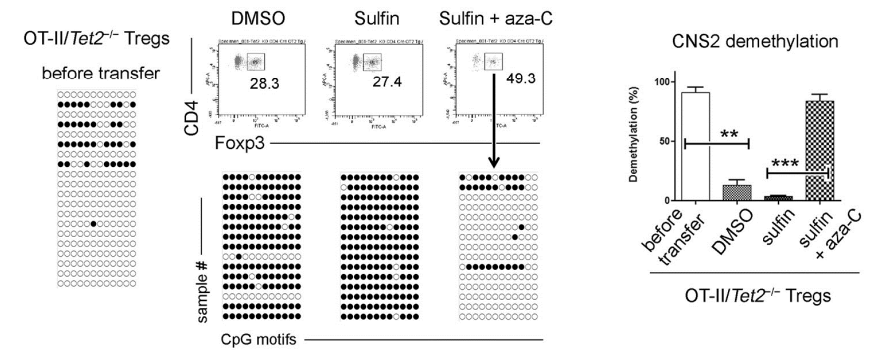

Fig. 1. Tet2 is essential to maintain CNS2 demethylation in Tregs under inflammatory conditions. (A) A schematic of the first part of the Foxp3 locus. White boxes indicate untranslated regions. Three CNS elements are shown in gray, and the locations of 10 individual $\mathrm{CpG}$ motifs within CNS2 are also shown. First CpG motif is located 4275 bases downstream of the TSS (transcription start site, +1). (B) FACSsorted WT or Tet2 ${ }^{-1-}$ OT-II Tregs (CD45.2 $2^{+}$) were injected into congenically marked WT B6 mice $\left(C D 45.1^{+}\right)$. Recipient mice were immun-

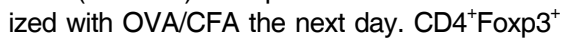
cells of donor origin were sorted from the draining LNs for the CNS2 demethylation study. Sorted cells were subjected to bisulfate sequencing for CNS2 analysis (right) and used to check the expression of Foxp3 (lower). (C-D) FACS-sorted WT or Tet2 ${ }^{-/}$OT-II Tregs were injected into WT B6 mice, which were immunized with OVA/CFA and treated with sulfinpyrazone (sulfin) or DMSO (vehicle) every day. $\mathrm{CD}^{+} \mathrm{Foxp}^{+}$cells of donor origin were resorted for the CNS2 demethylation assay. The extent of CNS2 demethylation of the donor Foxp3 $^{+}$cells (C) and the percentages of Foxp3 $^{+}$cells of donor $\mathrm{CD}^{+} \mathrm{T}$ cells (D) were statistically analyzed. Bars show the mean \pm SEM ( $n=6-8$, pooled from three independent experiments). (E) FACS-sorted Tet2 $2^{-/-}$OT-II Tregs were injected into WT B6 mice, which were immunized with OVA/CFA and additionally treated with sulfinpyrazone (sulfin) \pm 5 azacytidine (aza-C). Results of the CNS2 demethylation analysis from donor Tregs are shown (left). Results of the statistical analysis (right) are from a single experiment $(n=2$ mice/group, mean \pm SEM) representative of two independent experiments. Methylation status of 12 individual CpG motifs in the antisense strands of Foxp3 CNS2 is shown by white (demethylation) or black (methylation) circles. ${ }^{* \star} P<0.001 ;{ }^{* \star} P<0.01 ;{ }^{*} P<0.05$; NS, not significant.
Foxp3 stability and the suppressive function are impaired in Tet $2^{-1-}$ Tregs with methylated CNS2

The finding that CNS2 demethylation was labile in Tet2 ${ }^{-1-}$ Tregs was also seen in vitro. To recapitulate the inflammatory milieu, Tregs were cultured with anti-CD3 and CD28 mAbs (antiCD3/CD28) plus recombinant IL6 (rlL6) because IL6 is ubiquitously found in inflammatory conditions and has been shown to be associated with Foxp3 stability in Tregs (Feng et al., 2014). About $50 \%$ of the cells maintained the expression of Foxp3 in a 4-day-culture, and the Foxp $3^{+} C D 25^{+}$cells were re-sorted for CNS2 demethylation analysis (Fig. 2A, upper). Like the in vivo study, a significant number of $\mathrm{CpG}$ motifs were methylated in Tet2 $^{-1-}$ Tregs cultured in the presence of rlL6 (Fig. 2A, lower) implying that the methylation status of Foxp3 CNS2 is dynami- cally regulated and not fixed. We also performed the same experiments with rIL4 and rlL12; however, much less CpG motifs were methylated in the Tregs treated with rIL4 or rIL12 (Supplementary Fig. S3).

Next, we investigated the effect of CNS2 re-methylation on the Foxp3 stability and the suppressive function of the Tregs. Foxp3-expressing cells from Tregs treated with rIL6 shown in Fig. $2 \mathrm{~A}$ were re-sorted and cultured with anti-CD3/CD28 plus rlL2, and the expression of Foxp3 was analyzed after 3 days. Foxp3 was maintained stably in all the Tregs except for the Tet $2^{-1-}$ Tregs pre-treated with rlL6 (Fig. 2B), which was consistent with the results obtained in the CNS2 demethylation study. Next, we did an in vitro suppression test to check the suppressive function of the corresponding Tregs. Cell division 


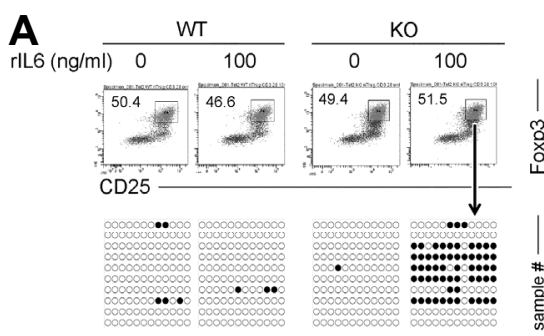

CPG motifs
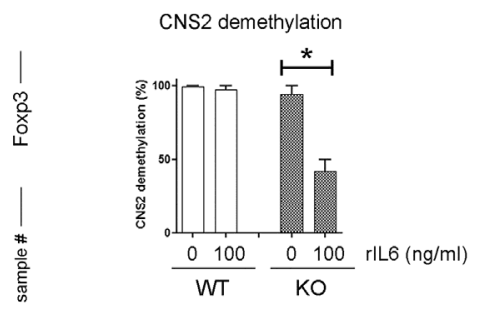

B
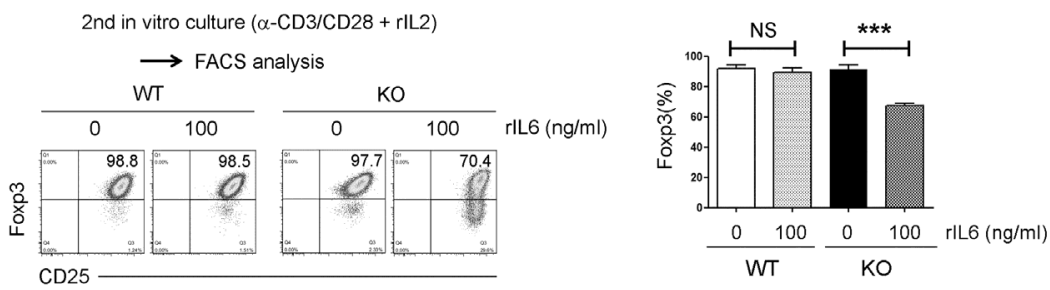

C

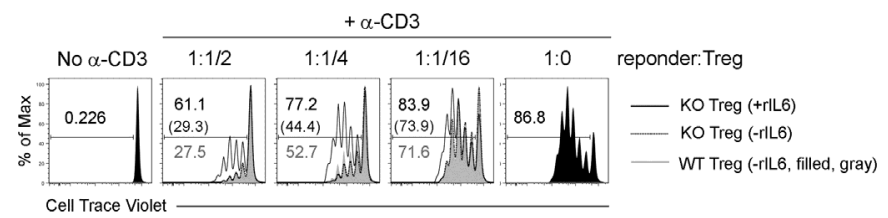

Fig. 2. The functions of Tregs is impaired by CNS2 re-methylation. (A) FACS-sorted WT or Tet $^{-/-}$Tregs were cultured with antiCD3/CD28 \pm rlL6 for 4 days. CD $4^{+}$Foxp3 ${ }^{+}$cells were re-sorted and used for the CNS2 demethylation study. Results of statistical analysis (right) are from a single experiment $(n=2-3$ samples/group, mean \pm SEM) representative of three independent experiments. (B) Foxp3 (GFP)-expressing Tregs isolated from (A) were cultured in the presence of anti-CD3/CD28 + rlL2 $(200 \mathrm{ng} / \mathrm{ml})$ and subjected to FACS analysis after 3 days. The frequency of Foxp $3^{+}$cells was statistically analyzed on the right side $(n=$ $5-6$, pooled from two independent experiments). (C) Cell division dye (cell trace violet)labeled responder cells were cultured in the presence of various Tregs sorted from (A). The levels of the cell division dye were checked after 3 days. Data are representative of three independent experiments. Methylation status of individual $\mathrm{CpG}$ motifs is shown by white (demethylation) or black (methylation) circles. Numbers in the indicated area in the FACS plots refer to the percentage of each subset. ${ }^{\star \star *} P<0.001 ;{ }^{*} P<0.05$; NS, not significant. dye (cell trace violet)-labeled $\mathrm{CD}^{+}{ }^{+}$responder cells were cocultured with antigen presenting cells, Tregs, and anti-CD3 mAbs, and the extent of proliferation of the responders was estimated with flow cytometry. Tet2 ${ }^{-1}$ Tregs pre-treated with rlL6 showed inferior suppressive activity compared to other Tregs (Fig. 2C) indicating that the methylation status of the CpG motifs in the Foxp3 CNS2 region is closely related with the Foxp3 expression and suppressive activity in Tregs.

IL2 is an important survival factor for Tregs (Malek, 2008), and media supplemented with rlL2 are commonly used to culture Tregs in vitro. Thus, we also did the CNS2 demethylation experiments with WT or Tet2 ${ }^{-1-}$ Tregs cultured in the presence of anti-CD3/CD28 + rIL6 plus rlL2. Unexpectedly, the CNS2 of the Tet $2^{-1-}$ Tregs was normal (Supplementary Fig. S4). These preliminary results led us to hypothesize whether IL2 reverses the effects of IL6 on CNS2 re-methylation.

IL2 prevents CNS2 methylation in a Tet-dependent manner Because IL2 could be produced by CD4 ${ }^{+}$Foxp $3^{-}$cells converted from Tregs and already available even without adding rIL2, we started to examine the potential roles of IL2 with a loss-offunction approach. To neutralize IL2, FACS-sorted WT or Tet $2^{-1-}$ Tregs were cultured with anti-IL2 blocking monoclonal antibodies (mAbs, clone: S4B6), which reduced the frequency of Foxp3 ${ }^{+}$cells and the expression level of CD25 (Fig. 3A). These findings were consistent with our idea that IL2 did already influence the remaining Tregs. Foxp $3^{+}$cells were resorted and subjected to bisulfite sequencing. The CNS2 demethylation analysis revealed that most of the CpG motifs became methylated by rlL6 in both the WT and Tet2 ${ }^{-/-}$when IL2 was neutralized (Figs. $3 \mathrm{~A}$ and $3 \mathrm{~B}$ ), and they were restored (demethylated) in the absence of S4B6 (i.e. in the presence of IL2), which occurred less efficiently in the Tet $2^{-/-}$Tregs. These findings suggest that IL2 prevented IL6-mediated CNS2 remethylation, albeit partially, in a Tet2 dependent manner. Given the potential redundancy between the members of the Tet family (Han et al., 2015; Yang et al., 2015), we next investigated whether other Tet family members like Tet1 and Tet3 functioned as substitutes for Tet2 downstream of IL2. WT and Tet $2^{-1-}$ Tregs were transfected with small interference RNAs (siRNAs) targeting Tet1 and Tet3 together and subjected to a CNS2 demethylation study. RT-PCR assays revealed that the amounts of Tet 1 and Tet3 transcripts were specifically reduced in the Tet1/Tet3 siRNA-transfected cells (Fig. 3C). Subsequently, the transfected Tregs were cultured with various doses of rIL2. During the cell culture periods, no significant change in the cell numbers or expression of Foxp3 was found in the siRNAtransfected cells (Supplementary Fig. S5). Next, Foxp $3^{+}$CD $25^{+}$ cells were re-sorted (Supplementary Fig. S6) and subjected to bisulfite sequencing. Knockdown of Tet1 and Tet3 significantly prevented rIL2 from restoring CNS2 demethylation in the Tet2 ${ }^{-l-}$ Tregs (Fig. 3D). Furthermore, knockdown of Tet1 and Tet3 caused CNS2 methylation in the WT Tregs although quite mildly unless rlL2 was added. Taken together, these results clearly show that IL2 prevents IL6 from inducing CNS2 re-methylation with the Tet members.

Next, to verify the role of Dmnts in vitro, we cultured WT and Tet $2^{-1-}$ Tregs in the presence of 5-azacytidine. Like seen in the in vivo results (Fig. 1E), IL6-induced CNS2 re-methylation was abrogated by 5-azacytidine treatment in vitro (Supplementary Fig. S7). 
A

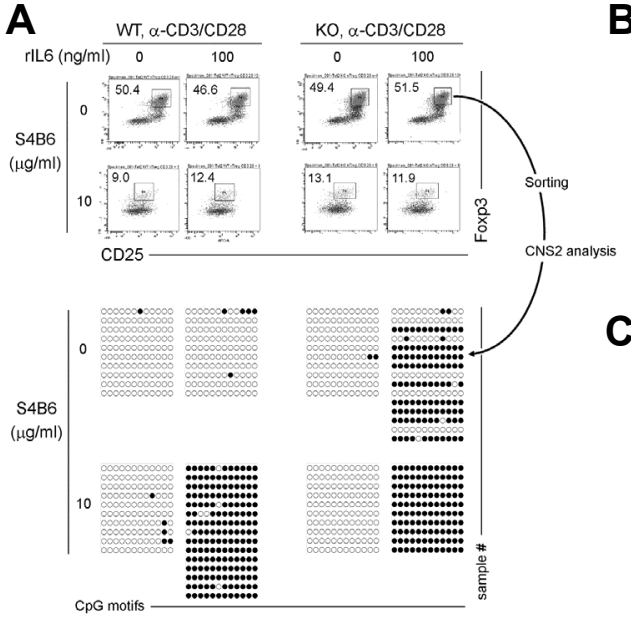

D

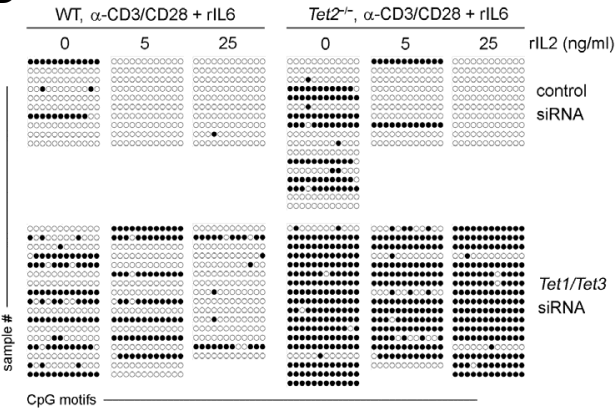

B

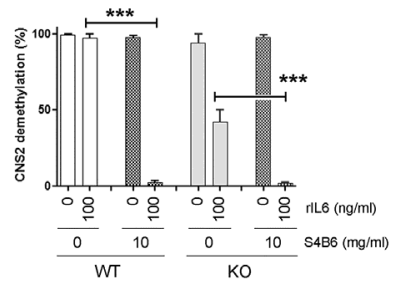

C
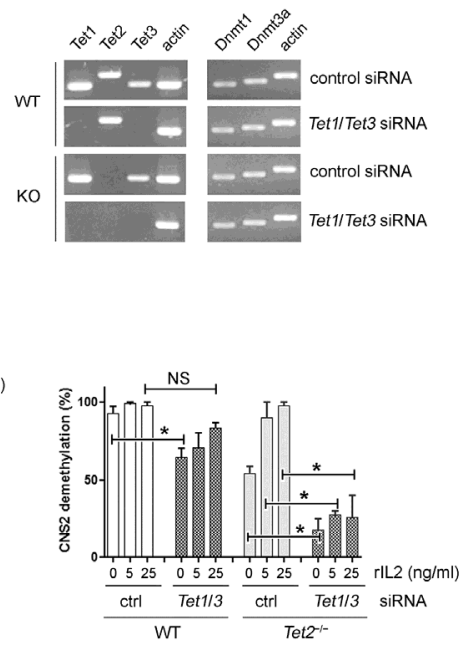

Fig. 3. IL2 inhibits CNS2 methylation in a Tetdependent manner. (A) FACS-sorted WT and Tet2 ${ }^{-/-}$Tregs were cultured under the indicated conditions and Foxp3 ${ }^{+}$cells were re-sorted for the CNS2 demethylation analysis. (B) Results of the statistical analysis of $(A)$ are shown as the mean \pm SEM of 2-3 samples/group (from one experiment representative of three independent experiments). (C) FACS-sorted WT and Tet2 $2^{--}$Tregs were transfected with Tet1/ Tet3 siRNAs, and the expression levels of the indicated gene transcripts were checked by RT-PCR. (D) WT and Tet2 ${ }^{-1-}$ Tregs transfected with Tet1/Tet3 siRNAs and cultured under the indicated conditions. Foxp $3^{+} \mathrm{CD} 25^{+}$cells were re-sorted (Supplementary Fig. S6) and subjected to bisulfate sequencing. Results of the statistical analysis are shown as the mean \pm SEM of 2-3 samples/group (from one experiment representative of two independent experiments). Methylation status of individual $\mathrm{CpG}$ motifs is shown by white (demethylation) or black (methylation) circles. Numbers in the indicated area in the FACS plots refer to the percentage of each subset. ${ }^{* \star} P<0.001$; ${ }^{\star} P<0.05$; NS, not significant.

\section{IL2 and vitamin C are required for CNS2 demethylation in vivo}

Subsequently, we investigated the role of IL2 on CNS2 demethylation with the OVA/CFA immunization model. FACSsorted OT-II/WT Tregs were injected into congenically marked WT B6 mice, which were treated with anti-IL2 blocking mAbs plus sulfinpyrazone. IL2 neutralization treatment downregulated CD25 in the donor and host Tregs (Supplementary Fig. S8) but did not cause a significant change in the CNS2 demethylation status. However, when sulfinpyrazone was treated together, more CpG motifs in the CNS2 locus became methylated (Figs. $4 \mathrm{~A}$ and $4 \mathrm{C}$ ), suggesting that vitamin $\mathrm{C}$ compensates for the loss of IL2 signals in Foxp3 CNS2 demethylation. Next, FACSsorted OT-II/Tet $2^{-1-}$ Tregs were injected together with OT-IINT naïve $C D 4^{+} \mathrm{T}$ cells at a $1 / 5$ ratio (Treg/naive) into WT B6 mice, which were treated with control (rat lgG) or anti-IL2 mAbs. CNS2 demethylation analysis revealed that CNS2 in the Tet2 ${ }^{-/}$ Tregs became demethylated when injected together with OT-II $\mathrm{CD}^{+} \mathrm{T}$ cells, and the effects of co-injection were abrogated by anti-IL2 mAbs (Figs. 4B and 4C). Altogether, these in vivo results were consistent with the in vitro results and imply again the essential roles of IL2 and Tet proteins in CNS2 demethylation.

Tet2 is recruited to the CNS2 locus by IL2 and hinders Dnmt1

It was reported that IL2 is related to the binding of Tet2 to Foxp3 enhancers like CNS1 and CNS2 (Yang et al., 2015), which led us to hypothesize that IL2 and IL6 would determine the methylation status of CNS2 by affecting the binding of Tet2 or Dnmts, respectively. To test this hypothesis, we examined the binding of Tet2 and Dnmt1 by chromatin immunoprecipitation followed by quantitative PCR (ChIP-qPCR) and compared their relative enrichments. Because it was recently proposed that both Dnmt1 and Dnmt3 are responsible together for CpG methylation of nascent and hemi-methylated DNA (Jeltsch and Jurkowska, 2014) and Dnmt1 is essential for Tregs (Josefowicz et al., 2009; Wang et al., 2013), Dnmt1 was selected in this experiment. FACS-sorted WT and Tet2 $2^{-1-}$ Tregs were cultured in the presence of anti-CD3/CD28 plus rlL6 \pm rlL2 for 3 days and used for the ChIP-qPCR analysis. Notably, Tet2 and Dnmt1 bound to the CNS2 locus in a mutually exclusive manner, which suggested that Tet2 and Dnmt1 might interfere with each other's binding to the same locus (Fig. 5). The binding of Tet2 to CNS2 was changed by rIL6 and rlL2: it was high in the 'without cytokine treatment' condition, decreased after the rIL6 treatment, and restored when treated with both rlL6 and rIL2. Dnmt1 also bound to CNS2 but in the opposite way. Furthermore, Tet2 deficiency led to an increase in the Dnmt1 occupancy on the CNS2 locus, suggesting that Dnmt1 binding to CNS2 is inhibited by Tet2 recruited by IL2. In contrast, the binding of Tet2 to the promoter region was not changed by IL 6 or IL2, and the binding of Dnmt1 was not detected in the promoter region. No signal was detected in DNAs immunoprecipitated by the isotype control Abs (data not shown). Altogether, these results imply that IL2 helped Tet2 to bind to CNS2, which subsequently interfered with the binding of Dnmt1 and eventually prevented Dnmts from inducing CNS2 methylation. However, it is also possible that Tet2 inhibits CNS2 methylation by actively removing the methyl-group that Dnmts had added to the CpG 
A
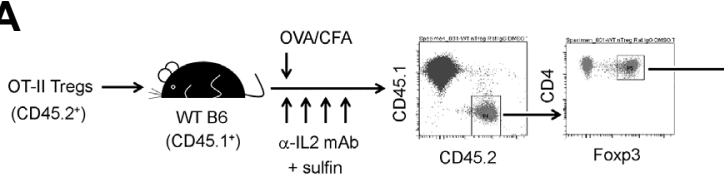

Rat

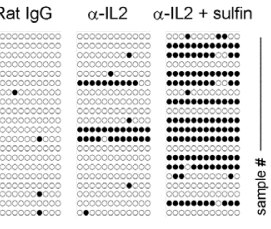

CPG motifs

B

B (CD $\left.45.2^{+}\right)$
T-II/Tet2--Tregs + OT-II naive $\left(\mathrm{CD} 45.1^{+} / 45.2^{+}\right)$
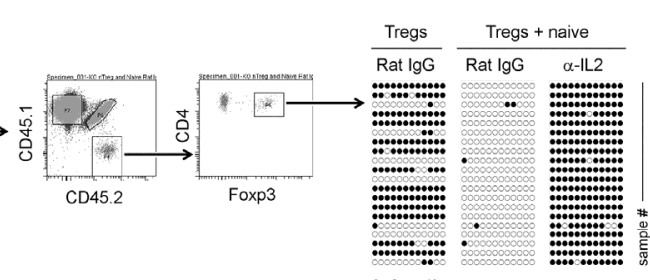

Fig. 4. IL2 and vitamin $C$ are required to prevent CNS2 re-methylation in vivo. (A-B) OTII/WT Tregs only (A) or OT-II/Tet2 ${ }^{-/-}$Tregs plus naïve OT-II T cells (B) were injected into WT mice, which were immunized with OVA/CFA and treated as indicated. $\mathrm{CD} 4^{+} \mathrm{Foxp}^{+}$cells of donor origin were re-sorted and used for the CNS2 demethylation assay. (C) Statistical analysis of the above CNS2 demethylation results $(A, B)$ is shown as the mean \pm SEM of 2-3 recipient mice/group (from one experiment representative of two independent experiments). Methylation status of individual CpG motifs is shown by white (demethylation) or black (methylation) circles. Numbers in the indicated area in the FACS plots refer to the percentage of each subset. ${ }^{* \star *} P<0.001 ;{ }^{* *} P<$ 0.01 ; NS, not significant.

C

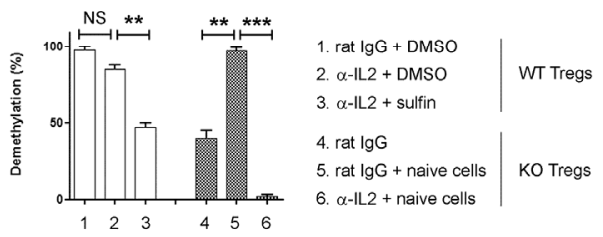

Tet2

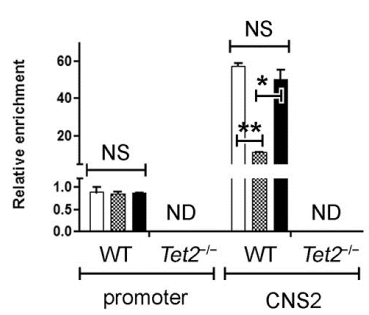

Dnmt1

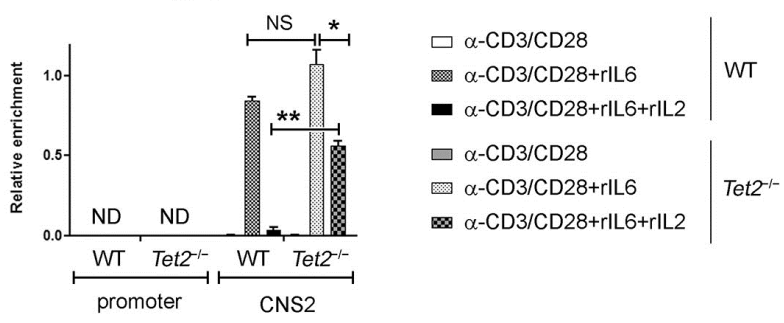

Fig. 5. Tet2 is recruited to CNS2 and inhibits the binding of Dnmt1. ChIP-qPCR of Tet2 and Dnmt1 at the Foxp3 promoter and CNS2 locus. FACS-sorted WT and Tet2 ${ }^{-/-}$Tregs were cultured in the presence of anti-CD3/CD28 plus rlL6 \pm rlL2, and used for the ChIP analysis. Subsequent qPCR was done on DNA precipitated by anti-Tet2 or anti-Dnmt1 Abs (mean \pm SEM of duplicates, from one experiment representative of three independent experiments). ${ }^{\star \star} P<0.01 ;{ }^{*} P<0.05$; NS, not significant; ND, not detected.

motifs of CNS2. To evaluate whether the enzymatic activity of Tet2 is required to oppose the activity of Dnmts, we analyzed the CNS2 demethylation of Tet2 $2^{-1-}$ Tregs transfected with plasmids encoding WT or catalytically inactive $(\mathrm{H} \times \mathrm{D})$ mutant Tet2 (Ko et al., 2010).

The Tet2 ${ }^{-/-}$Tregs were co-transfected with WT or mutant Tet2 plasmids (myc-tagged) together with siRNAs targeting Tet1 and Tet3 to investigate the mechanism of action of Tet2 alone. About $40 \%$ of the transfected cells expressed Tet 2 proteins $4 \mathrm{~h}$ after transfection, and the amounts of Tet2 transcripts produced by the introduced plasmids were similar to each other (Fig. 6A). Then, we cultured the transfected cells under the indicated conditions and isolated Tregs co-expressing the Foxp3 and myc epitopes. CNS2 demethylation analysis revealed that all the CpG motifs were demethylated in both the vector- and Tet2 (both WT and mutant)-transfected Tregs unless rIL6 was added. However, treatment with rlL6 \pm rlL2 induced CNS2 methylation differentially. Like shown previously (Fig. 3D), a significant number of CpG motifs in the Tet2 $2^{-/-}$Tregs, in which the Tet1/ Tet3 was knock downed, were methylated after rIL6 treatment, which was not restored by rlL2. However, the CpG motifs in the Tregs transfected with the WT Tet2 plasmids were much less methylated by rIL6 and almost completely demethylated by the addition of rIL6 + rIL2. Interestingly, the demethylation status of the Tet2 mutant Tregs was in between (Fig. 6B) indicating that Tet2 prevents CNS2 re-methylation under inflammatory conditions by both the DNA demethylase activity and by antagonizing the binding of Dnmts to CNS2. These results raised a question whether CNS2 demethylation would be restored in Tregs simply by enhancing the DNA demethylase activity of the Tet enzymes. To address this issue, FACS-sorted WT and Tet2 ${ }^{-/-}$ Tregs were cultured in the presence of anti-CD3/CD28 + rlL6 + S6B6 to induce CNS2 methylation, and vitamin C $(10 \mu \mathrm{g} / \mathrm{ml})$, a Tet agonist, was added only to a test group. Notably, vitamin C 
A

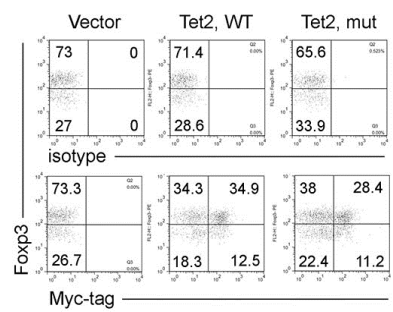

Tet1

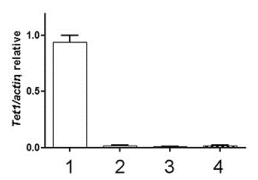

1. WT, control siRNA

2. Vector transfection

3. WT Tet2 transfection $\quad$ Tet2-1-, Tet1/3 siRNA

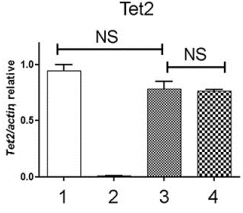

Tet3

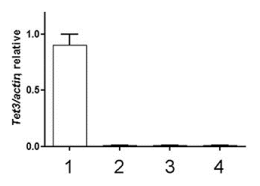

4. Mutant Tet2 transfection

B
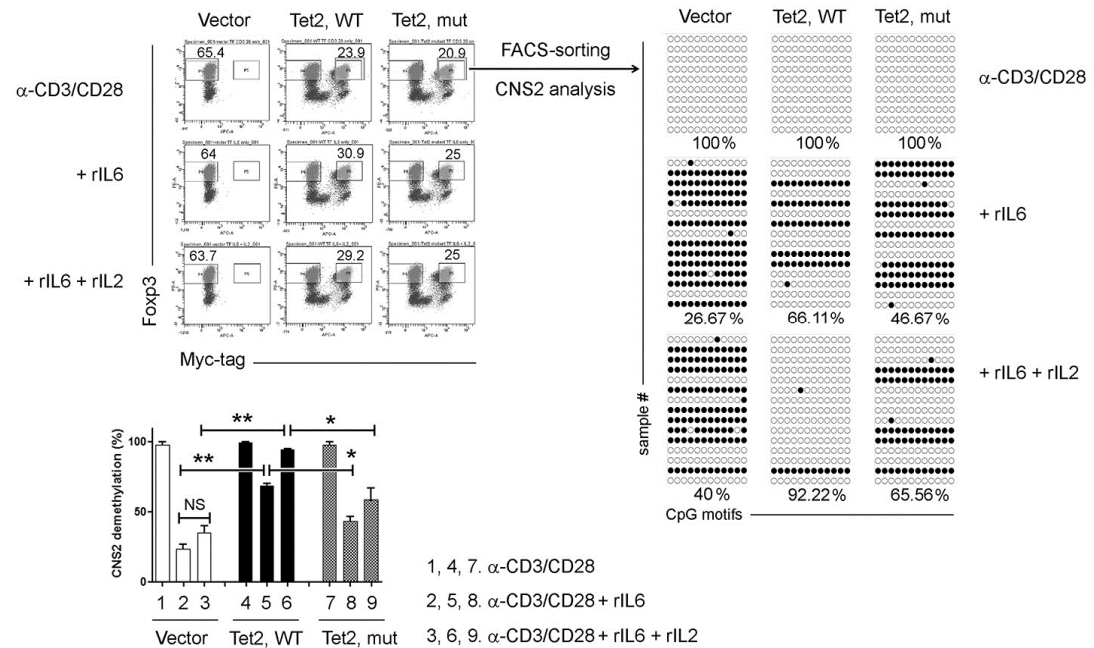

C

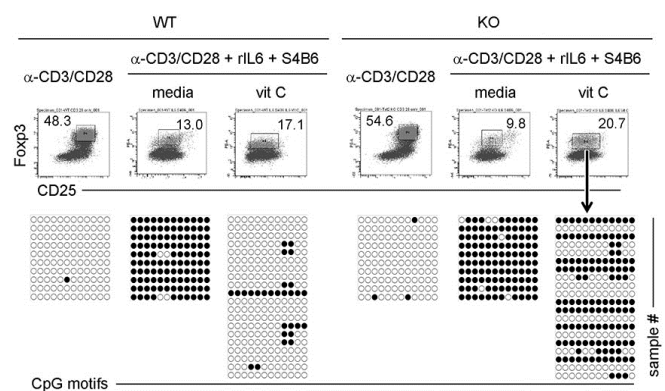

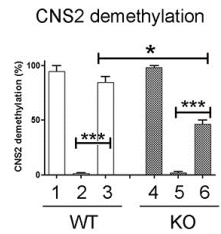

1, $4 . \alpha-\mathrm{CD} 3 / \mathrm{CD} 28$

2, 5. $\alpha-C D 3 / C D 28+r l L 6+S 4 B 6$

3, 6. $\alpha-\mathrm{CD} 3 / \mathrm{CD} 28+\mathrm{rlL} 6+\mathrm{S} 4 \mathrm{~B} 6+$ vit $\mathrm{C}$

Fig. 6. DNA demethylase activity of Tet2 is required to maintain CNS2 demethylation. (A) FACS-sorted Tet2 ${ }^{-1-}$ Tregs were co-transfected with plasmids encoding WT or mutant Tet2 (myc-tagged) together with siRNAs targeting Tet1 and Tet3. The expressions of Foxp3 and introduced Tet2 (myc) were checked by FACS (left, FACS plots). The expression levels of Tet1, Tet2, and Tet3 transcripts are also shown on the right side (mean \pm SEM of duplicates). Tet2 primers cannot distinguish the endogenous and introduced Tet2 transcripts (WT, mutant). (B) Tet2 ${ }^{-1-}$ Tregs transfected with vector or Tet2 plasmids were cultured under the indicated conditions, and Foxp $3^{+} \mathrm{Myc}(\mathrm{Tet} 2)^{+}$cells were re-sorted for CNS2 demethylation. Results of the statistical analysis on CNS2 demethylation are shown (lower) as the mean \pm SEM of 2 samples/group (from one experiment representative of two independent experiments). (C) FACS-sorted WT and Tet2 ${ }^{-1-}$ Tregs were cultured under the indicated conditions, and Foxp3 ${ }^{+}$cells were re-sorted for CNS2 demethylation analysis. Results of the statistical analysis (right) are shown as the mean \pm SEM of 2-3 samples/group (from one experiment representative of two independent experiments). Methylation status of individual CpG motifs is shown by white (demethylation) or black (methylation) circles. Numbers in the indicated area in the FACS plots refer to the percentage of each subset. ${ }^{* \star} P<0.001 ;{ }^{* \star} P<0.01 ;{ }^{*} P<0.05$; NS, not significant.

reversed the effect of IL6 and S4B6 in a Tet2 dependent manner (Fig. 6C), supporting the idea that the DNA demethylase activity of Tets contributes to maintaining CNS2 demethylation in Tregs.
Here, we showed that demethylation of the CpG motifs in the Foxp3 CNS2 region is determined by the competition between the DNA modifying enzymes Dnmts and Tets which have opposite functions. Dnmt1 was recruited to the CNS2 locus by the 
inflammatory cytokine IL6 (Fig. 5) and methylated the CpG motifs (Fig. 2). In contrast, the methylation activity of Dnmt1 was abrogated by Tet2 recruited to CNS2 by IL2 (Fig. 5). These findings suggest that IL2-Tet2 and IL6-Dnmt1 compete for the same binding sites in the CNS2 locus as STAT3 and STAT5 do in the IL17 locus (Yang et al., 2011) and Tet2 inhibits Dnmt1 through the occupation of CNS2. Furthermore, the enzymatic activity of Tet2 also contributed to CNS2 demethylation. While the demethylation status of CNS2 was restored completely in Tet $2^{-1-}$ Tregs transfected with WT Tet2 plasmids, catalytically inactive Tet2 $(\mathrm{H} \times \mathrm{D}$ mutant) plasmids showed only partial effects (Fig. 6B). Consistent with this result, CNS2 re-methylation induced in Tregs treated with rlL6 and S4B6 was prevented by vitamin $\mathrm{C}$ in a Tet2 dependent manner (Fig. $6 \mathrm{C}$ ). These findings altogether indicate that the demethylation status of CNS2 is regulated at multiple levels, and Foxp3 expression could be maintained consistently unless the activity of Tet proteins is reduced significantly. Indeed, Tet2 ${ }^{-/}$Tregs with methylated CNS2 lost Foxp3 expression significantly, which led to the impairment of suppressive functions (Fig. 2C), suggesting that CNS2 demethylation is closely related with the Foxp3 expression and suppressive activity in Tregs.

In conclusion, our study showed that the methylation status of the Foxp3 CNS2 locus is dynamically regulated by a balance between two DNA modifying enzymes Dnmts and Tets and related cytokines, which influences the stability of Foxp3 expression in Tregs.

Note: Supplementary information is available on the Molecules and Cells website (www.molcells.org).

\section{ACKNOWLEDGMENTS}

We thank Hyun-Ah Lee (Central laboratory Kangwon National University, Korea) for cell sorting and Divya Chandrasekharan Nair for genotyping. This work was supported by the National Research Foundation of Korea (NRF-2013R1A1A2059821) and Hallym University (HRF-201601-017).

\section{REFERENCES}

Burzyn, D., Kuswanto, W., Kolodin, D., Shadrach, J.L., Cerletti, M., Jang, Y., Sefik, E., Tan, T.G., Wagers, A.J., Benoist, C., et al. (2013). A special population of regulatory $T$ cells potentiates muscle repair. Cell 155, 1282-1295.

Campbell, D.J., and Koch, M.A. (2011). Phenotypical and functional specialization of FOXP3+ regulatory T cells. Nat. Rev. Immunol. $11,119-130$

Chaudhry, A., Rudra, D., Treuting, P., Samstein, R.M., Liang, Y., Kas, A., and Rudensky, A.Y. (2009). CD4+ regulatory T cells control TH17 responses in a Stat3-dependent manner. Science 326, 986-991.

Chung, Y., Tanaka, S., Chu, F., Nurieva, R.I., Martinez, G.J., Rawal, S., Wang, Y.H., Lim, H., Reynolds, J.M., Zhou, X.H., et al. (2011). Follicular regulatory T cells expressing Foxp3 and Bcl-6 suppress germinal center reactions. Nat. Med. 17, 983-988.

Cipolletta, D., Feuerer, M., Li, A., Kamei, N., Lee, J., Shoelson, S.E., Benoist, C., and Mathis, D. (2012). PPAR-gamma is a major driver of the accumulation and phenotype of adipose tissue Treg cells. Nature 486, 549-553.

Dickson, K.M., Gustafson, C.B., Young, J.I., Zuchner, S., and Wang, G. (2013). Ascorbate-induced generation of 5hydroxymethylcytosine is unaffected by varying levels of iron and 2-oxoglutarate. Biochem. Biophys. Res. Commun. 439, 522-527.

Feng, Y., Arvey, A., Chinen, T., van der Veeken, J., Gasteiger, G., and Rudensky, A.Y. (2014). Control of the inheritance of regulatory $T$ cell identity by a cis element in the Foxp3 locus. Cell $158,749-763$.

Floess, S., Freyer, J., Siewert, C., Baron, U., Olek, S., Polansky, J., Schlawe, K., Chang, H.D., Bopp, T., Schmitt, E., et al. (2007).
Epigenetic control of the foxp3 locus in regulatory T cells. PLoS Biol. 5, e38.

Fontenot, J.D., Gavin, M.A., and Rudensky, A.Y. (2003). Foxp3 programs the development and function of CD4+CD25+ regulatory T cells. Nat. Immunol. 4, 330-336.

Han, J.A., An, J., and Ko, M. (2015). Functions of TET proteins in hematopoietic transformation. Mol. Cells 38, 925-935.

Hansmann, L., Schmidl, C., Boeld, T.J., Andreesen, R., Hoffmann, P., Rehli, M., and Edinger, M. (2010). Isolation of intact genomic DNA from FOXP3-sorted human regulatory $T$ cells for epigenetic analyses. Eur. J. Immunol. 40, 1510-1512.

Hori, S., Nomura, T., and Sakaguchi, S. (2003). Control of regulatory $\mathrm{T}$ cell development by the transcription factor Foxp3. Science 299, 1057-1061.

Huehn, J., and Beyer, M. (2015). Epigenetic and transcriptional control of Foxp3+ regulatory T cells. Semin. Immunol. 27, 10-18.

Huehn, J., Polansky, J.K., and Hamann, A. (2009). Epigenetic control of FOXP3 expression: the key to a stable regulatory T-cell lineage? Nat. Rev. Immunol. 9, 83-89.

Jeltsch, A., and Jurkowska, R.Z. (2014). New concepts in DNA methylation. Trends Biochem. Sci. 39, 310-318.

Josefowicz, S.Z., Wilson, C.B., and Rudensky, A.Y. (2009). Cutting edge: TCR stimulation is sufficient for induction of Foxp3 expression in the absence of DNA methyltransferase 1 . J. Immunol. 182, 6648-6652.

Kim, H.P., and Leonard, W.J. (2007). CREB/ATF-dependent T cell receptor-induced FoxP3 gene expression: a role for DNA methylation. J. Exp. Med. 204, 1543-1551.

Kim, K.Y., Lee, G., Yoon, M., Cho, E.H., Park, C.S., and Kim, M.G. (2015). Expression analyses revealed thymic stromal cotransporter/Slc46A2 is in stem cell populations and is a putative tumor suppressor. Mol. Cells 38, 548-561.

Ko, M., Huang, Y., Jankowska, A.M., Pape, U.J., Tahiliani, M., Bandukwala, H.S., An, J., Lamperti, E.D., Koh, K.P., Ganetzky, R., et al. (2010). Impaired hydroxylation of 5-methylcytosine in myeloid cancers with mutant TET2. Nature 468, 839-843.

Koch, M.A., Tucker-Heard, G., Perdue, N.R., Killebrew, J.R., Urdahl K.B., and Campbell, D.J. (2009). The transcription factor T-bet controls regulatory $\mathrm{T}$ cell homeostasis and function during type 1 inflammation. Nat. Immunol. 10, 595-602.

Li, X., Liang, Y., LeBlanc, M., Benner, C., and Zheng, Y. (2014). Function of a Foxp3 cis-element in protecting regulatory $\mathrm{T}$ cell identity. Cell 158, 734-748.

Linterman, M.A., Pierson, W., Lee, S.K., Kallies, A., Kawamoto, S., Rayner, T.F., Srivastava, M., Divekar, D.P., Beaton, L., Hogan, J.J., et al. (2011). Foxp3+ follicular regulatory T cells control the germinal center response. Nat. Med. 17, 975-982.

Malek, T.R. (2008). The biology of interleukin-2. Annu. Rev. Immunol. 26, 453-479.

Mantei, A., Rutz, S., Janke, M., Kirchhoff, D., Jung, U., Patzel, V., Vogel, U., Rudel, T., Andreou, I., Weber, M., et al. (2008). siRNA stabilization prolongs gene knockdown in primary T lymphocytes. Eur. J. Immunol. 38, 2616-2625.

Miyao, T., Floess, S., Setoguchi, R., Luche, H., Fehling, H.J., Waldmann, H., Huehn, J., and Hori, S. (2012). Plasticity of Foxp3(+) T cells reflects promiscuous Foxp3 expression in conventional $\mathrm{T}$ cells but not reprogramming of regulatory $\mathrm{T}$ cells. Immunity 36, 262-275.

Moran-Crusio, K., Reavie, L., Shih, A., Abdel-Wahab, O., NdiayeLobry, D., Lobry, C., Figueroa, M.E., Vasanthakumar, A., Patel, J., Zhao, X., et al. (2011). Tet2 loss leads to increased hematopoietic stem cell self-renewal and myeloid transformation. Cancer Cell 20, 11-24.

Ohkura, N., Hamaguchi, M., Morikawa, H., Sugimura, K., Tanaka, A., Ito, Y., Osaki, M., Tanaka, Y., Yamashita, R., Nakano, N., et al. (2012). T cell receptor stimulation-induced epigenetic changes and Foxp3 expression are independent and complementary events required for Treg cell development. Immunity 37, 785-799.

Ohnmacht, C., Park, J.H., Cording, S., Wing, J.B., Atarashi, K., Obata, Y., Gaboriau-Routhiau, V., Marques, R., Dulauroy, S., Fedoseeva, M., et al. (2015). MUCOSAL IMMUNOLOGY. The microbiota regulates type 2 immunity through RORgammat(+) T cells. Science 349, 989-993.

Piper, C., Pesenacker, A.M., Bending, D., Thirugnanabalan, B. Varsani, H., Wedderburn, L.R., and Nistala, K. (2014). T cell expression of granulocyte-macrophage colony-stimulating factor 
in juvenile arthritis is contingent upon Th17 plasticity. Arthritis Rheumatol. 66, 1955-1960.

Polansky, J.K., Kretschmer, K., Freyer, J., Floess, S., Garbe, A., Baron, U., Olek, S., Hamann, A., von Boehmer, H., and Huehn, J. (2008). DNA methylation controls Foxp3 gene expression. Eur. J. Immunol. 38, 1654-1663.

Sakaguchi, S., Vignali, D.A., Rudensky, A.Y., Niec, R.E., and Waldmann, H. (2013). The plasticity and stability of regulatory $T$ cells. Nat. Rev. Immunol. 13, 461-467.

Sasidharan Nair, V., Song, M.H., and Oh, K.I. (2016). Vitamin C Facilitates Demethylation of the Foxp3 Enhancer in a TetDependent Manner. J. Immunol. 196, 2119-2131.

Sefik, E., Geva-Zatorsky, N., Oh, S., Konnikova, L., Zemmour, D., McGuire, A.M., Burzyn, D., Ortiz-Lopez, A., Lobera, M., Yang, J., et al. (2015). MUCOSAL IMMUNOLOGY. Individual intestinal symbionts induce a distinct population of RORgamma(+) regulatory T cells. Science 349, 993-997.

Toker, A., Engelbert, D., Garg, G., Polansky, J.K., Floess, S., Miyao, T., Baron, U., Duber, S., Geffers, R., Giehr, P., et al. (2013). Active demethylation of the Foxp3 locus leads to the generation of stable regulatory T cells within the thymus. J. Immunol. 190, 3180-3188.

Wang, Y., Su, M.A., and Wan, Y.Y. (2011). An essential role of the transcription factor GATA-3 for the function of regulatory T cells. Immunity 35, 337-348.

Wang, L., Liu, Y., Beier, U.H., Han, R., Bhatti, T.R., Akimova, T., and Hancock, W.W. (2013). Foxp3+ T-regulatory cells require DNA methyltransferase 1 expression to prevent development of lethal autoimmunity. Blood 121, 3631-3639.

Wieczorek, G., Asemissen, A., Model, F., Turbachova, I., Floess, S. Liebenberg, V., Baron, U., Stauch, D., Kotsch, K., Pratschke, J., et al. (2009). Quantitative DNA methylation analysis of FOXP3 as a new method for counting regulatory $T$ cells in peripheral blood and solid tissue. Cancer Res. 69, 599-608.

Yang, X.P., Ghoreschi, K., Steward-Tharp, S.M., RodriguezCanales, J., Zhu, J., Grainger, J.R., Hirahara, K., Sun, H.W., Wei, L., Vahedi, G., et al. (2011). Opposing regulation of the locus encoding IL-17 through direct, reciprocal actions of STAT3 and STAT5. Nat. Immunol. 12, 247-254.

Yang, R., Qu, C., Zhou, Y., Konkel, J.E., Shi, S., Liu, Y., Chen, C., Liu, S., Liu, D., Chen, Y., et al. (2015). Hydrogen Sulfide Promotes Tet1- and Tet2-Mediated Foxp3 Demethylation to Drive Regulatory T Cell Differentiation and Maintain Immune Homeostasis. Immunity 43, 251-263.

Yue, X., Trifari, S., Aijo, T., Tsagaratou, A., Pastor, W.A., ZepedaMartinez, J.A., Lio, C.W., Li, X., Huang, Y., Vijayanand, P., et al. (2016). Control of Foxp3 stability through modulation of TET activity. J. Exp. Med. 213, 377-397.

Zheng, Y., Josefowicz, S., Chaudhry, A., Peng, X.P., Forbush, K., and Rudensky, A.Y. (2010). Role of conserved non-coding DNA elements in the Foxp3 gene in regulatory T-cell fate. Nature 463 808-812. 